\title{
Graphs with four boundary vertices
}

\author{
Tobias Müller* Attila Pór ${ }^{\dagger} \quad$ Jean-Sébastien Sereni $^{\ddagger}$ \\ Submitted: Feb 17, 2009; Accepted: Dec 17, 2010; Published: Jan 5, 2011 \\ Mathematics Subject Classification: 05C75
}

\begin{abstract}
A vertex $v$ of a graph $G$ is a boundary vertex if there exists a vertex $u$ such that the distance in $G$ from $u$ to $v$ is at least the distance from $u$ to any neighbour of $v$. We give a full description of all graphs that have exactly four boundary vertices, which answers a question of Hasegawa and Saito. To this end, we introduce the concept of frame of a graph. It allows us to construct, for every positive integer $b$ and every possible "distance-vector" between $b$ points, a graph $G$ with exactly $b$ boundary vertices such that every graph with $b$ boundary vertices and the same distance-vector between them is an induced subgraph of $G$.
\end{abstract}

\section{Introduction}

Let $G=(V, E)$ be a graph. A vertex $v \in V$ is a boundary vertex of $G$ if there exists a vertex $u \in V$ such that $\mathrm{d}(u, v) \geq \mathrm{d}(u, w)$ for all neighbours $w$ of $v$. Such a vertex $u$ is then called a witness for $v$. The boundary of $G$ is the set $\mathscr{B}(G)$ of boundary vertices of $G$.

The notion of boundary of a graph was introduced by Chartrand et al. [2,3] and studied further by Cáceres et al. [1], Hernando et al. [5], and Hasegawa and Saito [4]. In a short note [6], we gave a tight bound (up to a constant factor) on the order of the boundary of a graph in function of its maximum (or minimum) degree, thereby settling a problem suggested by Hasegawa and Saito [4].

*School of Mathematical Sciences, Sackler Faculty of Exact Sciences, Tel Aviv University, Tel Aviv 69978, Israel. E-mail: tobias@post.tau.ac.il. Research partially supported by an ERC advanced grant.

$\dagger$ Department of Mathematics, Western Kentucky University, Bowling Green, KY 42101, USA. E-mail: attila.por@wku.edu.

${ }^{\ddagger}$ CNRS (LIAFA, Université Denis Diderot), Paris, France and Department of Applied Mathematics (KAM) - Faculty of Mathematics and Physics, Charles University, Prague, Czech Republic. E-mail: sereni@kam.mff.cuni.cz. This author's work was partially supported by the European project IST FET AEOLus and the French Agence Nationale de la Recherche under reference ANR-10-JCJC-HEREDIA.

THE ELECTRONiC JOURNAL OF COMBINATORICS 18 (2011), \#P11 
Note that all vertices of a disconnected graph are boundary vertices. Hence we shall restrict attention to connected graphs in the rest of the paper. Every graph with more than one vertex has at least two boundary vertices, namely the endvertices of a longest path. As noted by Hasegawa and Saito [4], a connected graph has exactly two boundary vertices if and only if it is a path. In addition, they described all connected graphs with exactly three boundary vertices. Attaching a path $P$ to a vertex $v$ of a graph $G$ means taking the disjoint union of $G$ and $P$ and identifying $v$ with an end-vertex of $P$. A path of arbitrary length may have length 0 .

Theorem 1 (Hasegawa and Saito [4]). A connected graph $G$ has exactly three boundary vertices if and only if either

(i) $G$ is a subdivision of $K_{1,3}$; or

(ii) $G$ can be obtained from $K_{3}$ by attaching exaclty one path (of arbitrary length) to each of its vertices.

Hasegawa and Saito [4] asked for a characterisation of all graphs with four boundary vertices. The aim of the current paper is to provide such a characterisation. The statement of our main result requires a number of definitions and we therefore postpone it until the next section.

An important tool in our proof is the concept of the frame of a graph, which is of independent interest. The frame is the vector of all distances between the boundary vertices. In Section 3 we study frames in general. In particular, for every positive integer $b$ and every possible "distance-vector" between $b$ points, we explicitly construct a graph $F$ with exactly $b$ boundary vertices such that every graph with $b$ boundary vertices and the same distance-vector between them is an induced subgraph of $F$.

Let us note that Hasegawa and Saito [4] proved that any connected graph with exactly four boundary vertices has minimum degree at most 6 . Our description shows that the minimum degree is in fact never more than 3.

\section{Statement of the main result}

Before giving the description of all connected graphs with four boundary vertices, we need to introduce several definitions. The reader may find the next batch of definitions easier to digest by looking at figure 1 below.

Definition 2. Let $a$ and $c$ be two positive integers.

- The $(a \times c)$-grid is the graph $G_{a \times c}$ with vertex set

$$
V_{a \times c}^{0}:=\left\{(x, y) \in \mathbf{N}^{2} \mid 0 \leq x \leq a \text { and } 0 \leq y \leq c\right\}
$$

and an edge between vertices of Euclidean distance 1. Note that with our definition, the $(a \times c)$-grid has $(a+1) \cdot(c+1)$ vertices and not $a \cdot c$ as is customary. 
- The graph $N_{a \times c}$ has vertex set $V_{a \times c}:=V_{a \times c}^{0} \cup V_{a \times c}^{1}$, where

$$
V_{a \times c}^{1}:=\left\{\left(x+\frac{1}{2}, y+\frac{1}{2}\right) \mid(x, y) \in \mathbf{N}^{2}, 0 \leq x<a \text { and } 0 \leq y<c\right\} .
$$

There is an edge between two vertices if the Euclidean distance is at most 1.

- If $a>2$ then $X_{a \times c}$ is the subgraph of $N_{(a-1) \times c}$ induced by

$$
V_{(a-1) \times c} \backslash\left\{(x, y) \in \mathbf{N}^{2} \mid 0<x<a-1 \text { and } y \in\{0, c\}\right\} .
$$

If $a=2$ then $X_{2 \times c}$ is the subgraph of $N_{1 \times c}$ obtained by removing the edge between the vertices $(0,0)$ and $(1,0)$, and the edge between the vertices $(0, c)$ and $(1, c)$. If $a=1$ and $c>1$ then we take the same construction with $a$ and $c$ swapped, i.e. $X_{a \times c}:=X_{c \times a}$. Moreover, we let $X_{1 \times 1}$ be $K_{4}$, the complete graph on four vertices. Note that $X_{a \times c}$ is isomorphic to $X_{c \times a}$.

- The graph $T_{a \times c}$ is the subgraph of $N_{a \times(c+1)}$ induced by

$$
V_{a \times(c+1)} \backslash(\{(0, y) \mid y \in \mathbf{N}\} \cup\{(x, y) \mid x<a \text { and } y \in\{0, c+1\}\}) .
$$

- Let $G_{a \times c}^{1}$ and $G_{a \times c}^{2}$ be two copies of the $(a \times c)$-grid with vertex sets

$$
V_{1}:=\left\{v_{x, y} \mid 0 \leq x \leq a, 0 \leq y \leq c\right\} \text { and } V_{2}:=\left\{w_{x, y} \mid 0 \leq x \leq a, 0 \leq y \leq c\right\},
$$

respectively. The graph $D_{a \times c}$ is obtained from $G_{a \times c}^{1}$ and $G_{a \times c}^{2}$ by identifying $v_{x, y}$ with $w_{x, y}$ for all $x$ and $y$ such that $x \in\{0, a\}$ or $y \in\{0, c\}$; and adding an edge between $v_{x, y}$ and $w_{x, y}$ whenever $0<x<a$ and $0<y<c$, and an edge between $w_{x, y+1}$ and $v_{x+1, y}$ whenever $0 \leq x<a$ and $0 \leq y<c$.

- The graph $L_{a \times c}$ is obtained from $D_{a \times c}$ by removing the vertices $w_{x, y}$ for $x \in$ $\{1,2, \ldots, a-1\}$ and $y \in\{1,2, \ldots, c-1\}$.

It is straightforward to check that each of the graphs $N_{a \times c}, X_{a \times c}, T_{a \times c}, D_{a \times c}$ and $L_{a \times c}$ has exactly four boundary vertices.

Definition 3. A set $W \subseteq \mathbf{R}^{2}$ is axis slice convex if

- whenever both $\left(x_{1}, y\right)$ and $\left(x_{2}, y\right)$ belong to $W$ and $x_{1}<x_{2}$, then $\left\{(x, y) \mid x_{1} \leqslant x \leqslant x_{2}\right\} \subseteq W$; and

- whenever both $\left(x, y_{1}\right)$ and $\left(x, y_{2}\right)$ belong to $W$ and $y_{1}<y_{2}$, then $\left\{(x, y) \mid y_{1} \leqslant y \leqslant y_{2}\right\} \subseteq W$.

We are now in a position to state the characterisation of all connected graphs with four boundary vertices. Figure 2 provides examples of graphs from each of the nine families mentioned below. 

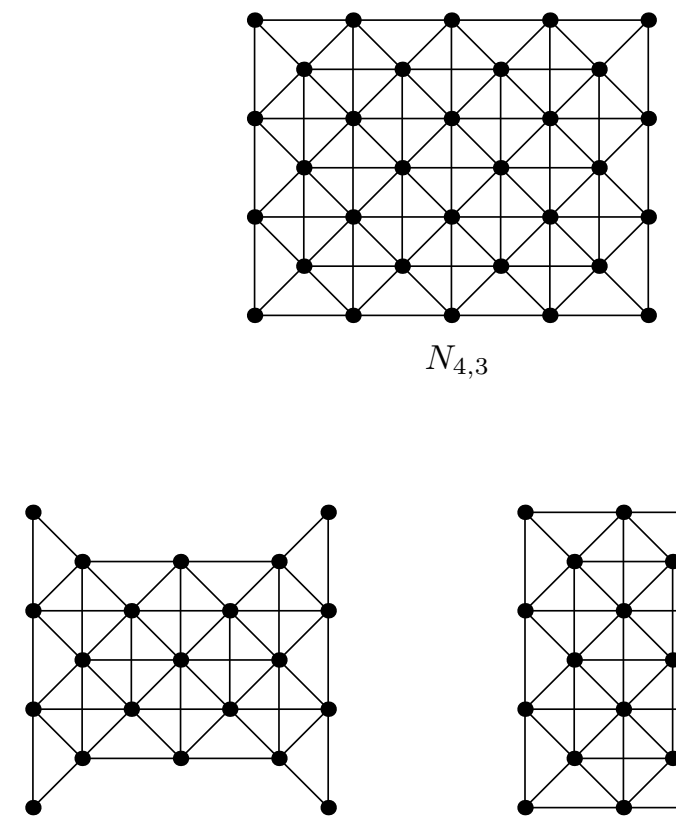

$X_{4,3}$

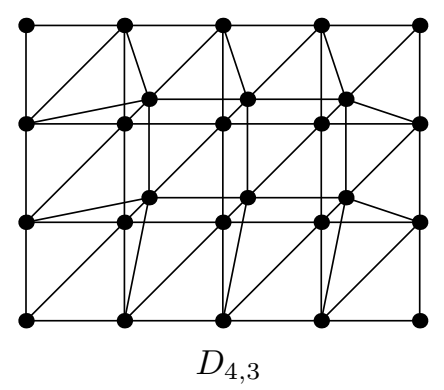

Figure 1: The graphs $N_{4 \times 3}, X_{4 \times 3}, T_{4 \times 3}, D_{4 \times 3}$ and $L_{4 \times 3}$. 
Theorem 4. A connected graph $G$ has exactly four boundary vertices if and only if it is either

(i) a subdivision of $K_{1,4}$; or

(ii) a subdivision of the tree with exactly four leaves and two vertices of degree 3; or

(iii) a graph obtained from one of the trees of (ii) by removing a vertex of degree 3 and adding all edges between its three neighbours; or

(iv) the complete graph $K_{4}$ on four vertices with exactly one path (of arbitrary length) attached to each of its vertices; or

(v) a subgraph of $N_{a \times c}$ induced by $V_{a \times c}^{0} \cup\left(W \cap V_{a \times c}^{1}\right)$ for some axis slice convex set $W \subseteq \mathbf{R}^{2}$, with exactly one path (of arbitrary length) attached to each of its boundary vertices; or

(vi) the graph $X_{a \times c}$ with exactly one path (of arbitrary length) attached to each of its boundary vertices; or

(vii) a subgraph of $T_{a \times c}$ induced by $V_{a \times(c+1)}^{1} \cup\left(W \cap V_{a \times(c+1)}^{0}\right)$ for some axis slice convex set $W \subseteq \mathbf{R}^{2}$ that contains $(a, 0)$ and $(a, c+1)$, with exactly one path (of arbitrary length) attached to each of its boundary vertices; or

(viii) the graph $D_{a \times c}$ with exactly one path (of arbitrary length) attached to each of its boundary vertices; or

(ix) the graph $L_{a \times c}$ with exactly one path (of arbitrary length) attached to each of its boundary vertices.

Our main tool in the proof of Theorem 4 is the frame of a graph, which we introduce and study next.

\section{The frame of a graph}

Definition 5. A frame is a metric space $(X, \mathrm{~d})$ where $X$ is a finite set and $d: X^{2} \rightarrow \mathbf{N}$ is an integer-valued distance function.

Let $G=(V, E)$ be a graph with boundary $\mathscr{B}:=\left\{B_{1}, \ldots, B_{b}\right\}$. The pair $\mathscr{F}(G):=$ $\left(\mathscr{B}, \mathrm{d}_{G}\right)$, where $\mathrm{d}_{G}$ is the distance in the graph $G$, is a frame. It is the frame of the graph $G$. For each vertex $v \in V$ we define the position vector

$$
\varphi(v):=\left(\mathrm{d}_{G}\left(v, B_{1}\right), \ldots, \mathrm{d}_{G}\left(v, B_{b}\right)\right),
$$

that represents its distances from the boundary vertices. For $x, y \in \mathbf{R}^{b}$, let $\mathrm{d}^{*}(x, y)$ be the $L_{\infty}$-distance between $x$ and $y$, i.e.

$$
\mathrm{d}^{*}(x, y):=\max _{1 \leqslant i \leqslant b}\left|x_{i}-y_{i}\right| .
$$



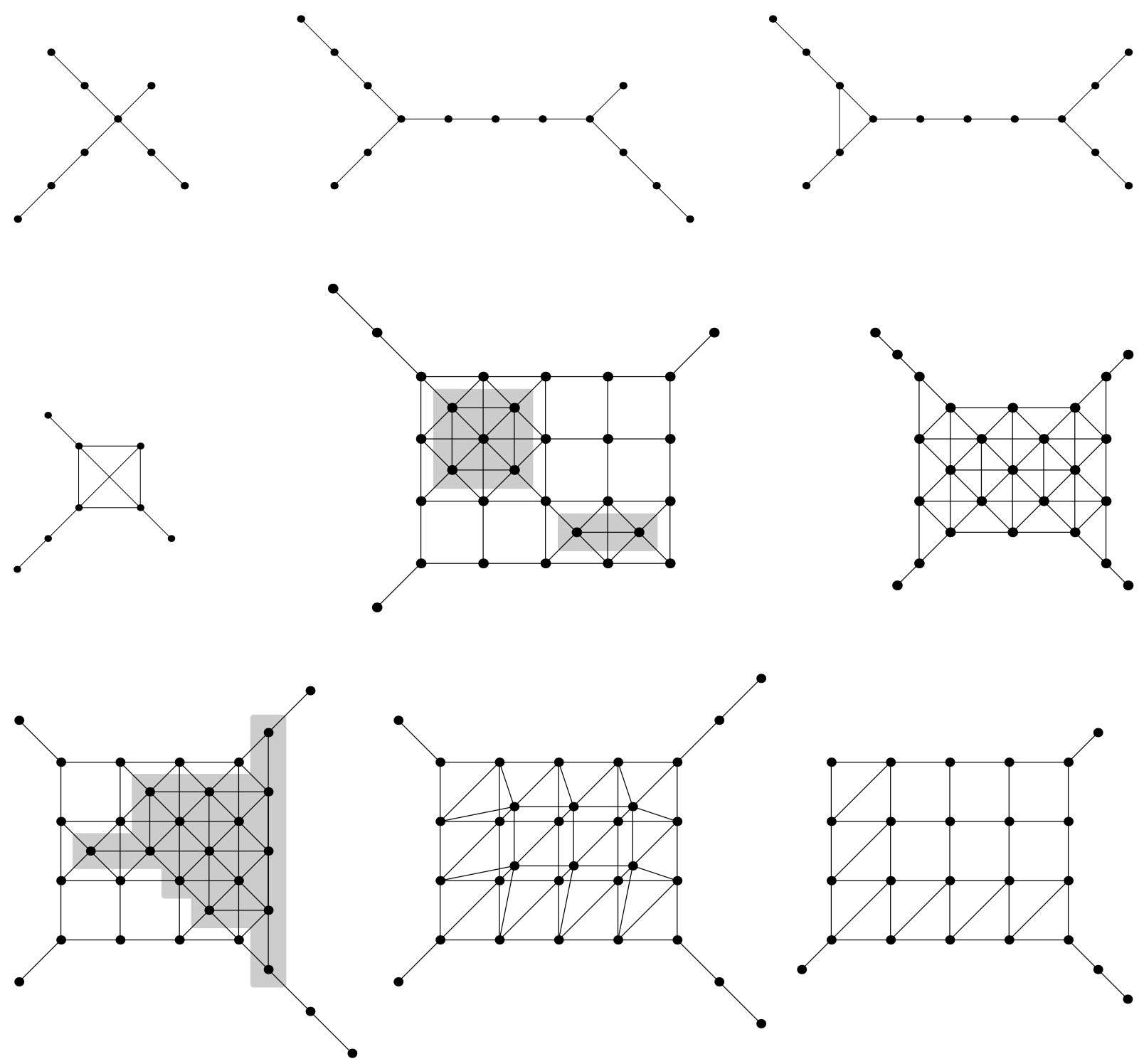

Figure 2: (Examples of) all types of connected graphs with 4 boundary vertices. The shading indicates the axis slice convex sets. 
Throughout the rest, we make use of the following observation [6, Lemma 3], which also appeared implicitly in former papers [2, 3].

Lemma 6. Each shortest path of $G$ extends to a shortest path between two boundary vertices.

We now prove that the $L_{\infty}$-distance of the position vectors of vertices of a graph is the same as their distance in the graph.

Lemma 7. $\mathrm{d}^{*}(\varphi(u), \varphi(v))=\mathrm{d}_{G}(u, v)$ for all $u, v \in V(G)$.

Proof of Lemma 7 . Let $\mathscr{B}:=\left\{B_{1}, \ldots, B_{b}\right\}$ be the boundary of $G$. Fix two vertices $u$ and $v$ of $G$. For each $i \in\{1,2, \ldots, b\}$,

$$
\varphi(u)_{i}=\mathrm{d}_{G}\left(u, B_{i}\right) \leq \mathrm{d}_{G}(u, v)+\mathrm{d}_{G}\left(v, B_{i}\right)=\mathrm{d}_{G}(u, v)+\varphi(v)_{i} .
$$

So $\mathrm{d}_{G}(v, u)=\mathrm{d}_{G}(u, v) \geq \varphi(u)_{i}-\varphi(v)_{i}$, and hence $\mathrm{d}_{G}(u, v) \geq\left|\varphi(u)_{i}-\varphi(v)_{i}\right|$. Therefore $\mathrm{d}_{G}(u, v) \geq \mathrm{d}^{*}(u, v)$.

By Lemma 6, any shortest path between $v$ and $u$ extends to a shortest path $P$ between two boundary vertices, say $B_{i}$ and $B_{j}$. If $B_{i}$ is the endvertex of $P$ closer to $u$ then

$$
\varphi(v)_{i}=\mathrm{d}_{G}\left(v, B_{i}\right)=\mathrm{d}_{G}(v, u)+\mathrm{d}_{G}\left(u, B_{i}\right)=\mathrm{d}_{G}(v, u)+\varphi(u)_{i} .
$$

Consequently, $\mathrm{d}_{G}(v, u)=\varphi(v)_{i}-\varphi(u)_{i} \leq \mathrm{d}^{*}(u, v)$, which finishes the proof.

The next lemma states two properties of the position vectors.

Lemma 8. Let $G=(V, E)$ be a graph with boundary $\mathscr{B}:=\left\{B_{1}, \ldots, B_{b}\right\}$. For every vertex $u \in V$, the position vector $\varphi(u)$ has the following properties.

(i) $\varphi(u)_{i}+\varphi(u)_{j} \geq \mathrm{d}_{G}\left(B_{i}, B_{j}\right)$ for every $i, j \in\{1, \ldots, b\}$; and

(ii) for every $i \in\{1, \ldots, b\}$, there exists $j \in\{1, \ldots, b\}$ such that $\varphi(u)_{i}+\varphi(u)_{j}=$ $\mathrm{d}_{G}\left(B_{i}, B_{j}\right)$.

Proof. Part (i) is a direct consequence of the triangle inequality:

$$
\varphi(u)_{i}+\varphi(u)_{j}=\mathrm{d}_{G}\left(u, B_{i}\right)+\mathrm{d}_{G}\left(u, B_{j}\right) \geq \mathrm{d}_{G}\left(B_{i}, B_{j}\right) .
$$

Part (ii) follows from Lemma 6 , since for every vertex $u$ and every boundary vertex $B_{i}$ there exists a shortest path between $B_{i}$ and $B_{j}$ containing $u$, for some index $j \in$ $\{1,2, \ldots, b\}$.

Definition 9. Suppose that $\mathscr{F}=(X, \mathrm{~d})$ is a frame with $X=\left\{B_{1}, \ldots, B_{b}\right\}$. We associate a graph $F=F(\mathscr{F})$ with the frame $\mathscr{F}$, called the frame-graph corresponding to $\mathscr{F}$. The vertex set $V(F)$ consists of the set of $b$-dimensional integer vectors $x=\left(x_{1}, \ldots, x_{b}\right) \in$ $\left(\mathbf{Z}^{+}\right)^{b}$ that satisfy

(F1) $x_{i}+x_{j} \geq \mathrm{d}\left(B_{i}, B_{j}\right)$ for every $(i, j) \in\{1,2, \ldots, b\}^{2}$; and 
(F2) for every $i \in\{1,2, \ldots, b\}$ there exists $j \in\{1,2, \ldots, b\}$ such that $x_{i}+x_{j}=\mathrm{d}\left(B_{i}, B_{j}\right)$.

If $x, y \in V(F)$ then $x y \in E(F)$ if and only if $\mathrm{d}^{*}(x, y)=1$.

For instance, if $X=\left\{B_{1}, B_{2}, B_{3}, B_{4}\right\}$ and the distance function is given by

$$
\begin{array}{lll}
\mathrm{d}\left(B_{1}, B_{2}\right)=2, & \mathrm{~d}\left(B_{1}, B_{3}\right)=3, & \mathrm{~d}\left(B_{1}, B_{4}\right)=1, \\
\mathrm{~d}\left(B_{2}, B_{3}\right)=2, & \mathrm{~d}\left(B_{2}, B_{4}\right)=3, & \mathrm{~d}\left(B_{3}, B_{4}\right)=2,
\end{array}
$$

then

$$
\begin{array}{r}
V(F)=\{(0,2,3,1),(1,1,2,2),(1,2,2,1),(1,3,2,0), \\
(2,0,2,3),(2,1,1,2),(2,2,1,1),(3,2,0,2)\}
\end{array}
$$

and $F$ is isomorphic to $T_{2 \times 1}$ (see Figure 3$)$.

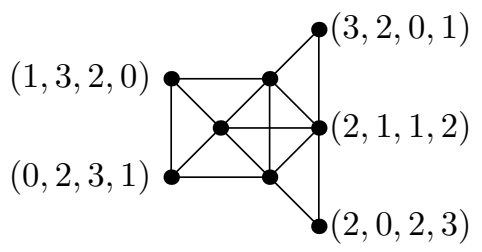

Figure 3: A frame-graph.

Note that the vector $\varphi\left(B_{k}\right)=\left(\mathrm{d}\left(B_{k}, B_{1}\right), \ldots, \mathrm{d}\left(B_{k}, B_{b}\right)\right)$ is in $V(F)$ for $k \in\{1, \ldots, b\}$.

We state and prove three lemmas. Recall that $\mathrm{d}^{*}(x, y):=\max _{i}\left|x_{i}-y_{i}\right|$ is the $L_{\infty^{-}}$ distance between $x$ and $y$.

Lemma 10. $\mathrm{d}_{F}(x, y)=\mathrm{d}^{*}(x, y)$ for all $x, y \in V(F)$.

Proof. Let $x$ and $y$ be two vertices of $F$ and set $t:=\mathrm{d}^{*}(x, y)$. Since there is a coordinate in which $x$ and $y$ differ by $t$, it follows from the definition of $F$ that $\mathrm{d}_{F}(x, y) \geq t$.

We now show that $\mathrm{d}_{F}(x, y) \leq \mathrm{d}^{*}(x, y)$ for any two vertices $x$ and $y$ by induction on $t:=\mathrm{d}^{*}(x, y) \geqslant 0$. If $t=0$ then $x_{i}=y_{i}$ for all $i$, so $x=y$. If $t=1$ then $x y \in E(F)$ by the definition of $F$, and hence $\mathrm{d}_{F}(x, y)=1$. If $t>1$ then we show that there exists a vector $x^{\prime} \in V(F)$ such that $x x^{\prime} \in E(F)$ and $\mathrm{d}^{*}\left(x^{\prime}, y\right)<t$. This yields the desired result, because by the induction hypothesis $\mathrm{d}_{F}\left(x^{\prime}, y\right)<t$, and therefore $\mathrm{d}_{F}(x, y) \leq t$.

For every $i \in\{1,2, \ldots, b\}$, let $x_{i}^{\prime}$ be the smallest of $x_{i}-1, x_{i}$ and $x_{i}+1$ that satisfies $x_{i}^{\prime}+x_{j}^{\prime} \geq \mathrm{d}\left(B_{i}, B_{j}\right)$ for every $j<i$ and $\left|x_{i}^{\prime}-y_{i}\right|<t$. First, we need to show that at least one of $x_{i}-1, x_{i}$ and $x_{i}+1$ satisfies the last two conditions.

Assume that $x_{1}^{\prime}, \ldots, x_{i-1}^{\prime}$ are defined for some integer $i \geqslant 1$. The choice $x_{i}^{\prime}=x_{i}+1$ ensures that $x_{i}^{\prime}+x_{j}^{\prime} \geq\left(x_{i}+1\right)+\left(x_{j}-1\right)=x_{i}+x_{j} \geq \mathrm{d}\left(B_{i}, B_{j}\right)$ by (F1). If $\left|x_{i}+1-y_{i}\right|<t$ then $x_{i}+1$ is a possible choice. If $\left|x_{i}+1-y_{i}\right| \geq t$ then let $x_{i}^{\prime}=y_{i}+t-1$. Since $x_{i} \leq y_{i}+t$, it follows that $x_{i}-1 \leq x_{i}^{\prime} \leq x_{i}$. As $t \geqslant 2$, for every $j<i$

$$
x_{j}^{\prime}+x_{i}^{\prime} \geq y_{j}-(t-1)+y_{i}+t-1=y_{j}+y_{i} \geq \mathrm{d}\left(B_{i}, B_{j}\right) .
$$


Therefore, $y_{i}+t-1 \in\left\{x_{i}-1, x_{i}\right\}$ is a possible choice.

Now we show that $x^{\prime} \in V(F)$. By the definition, $x_{i}^{\prime}+x_{j}^{\prime} \geq \mathrm{d}\left(B_{i}, B_{j}\right)$. If $x_{i}^{\prime}=x_{i}-1$ then since $x \in V(F)$ there exists $j$ such that $x_{i}+x_{j}=\mathrm{d}\left(B_{i}, B_{j}\right)$. So $x_{i}^{\prime}+x_{j}^{\prime} \leq\left(x_{i}-1\right)+\left(x_{j}+1\right)=$ $\mathrm{d}\left(B_{i}, B_{j}\right)$. If $x_{i}^{\prime}>x_{i}-1$ then $x_{i}^{\prime}-1$ was not a possible choice. Thus, either there exists $j<i$ such that $x_{j}^{\prime}+x_{i}^{\prime}-1<\mathrm{d}\left(B_{j}, B_{i}\right)$ in which case $x_{j}^{\prime}+x_{i}^{\prime} \leq \mathrm{d}\left(B_{i}, B_{j}\right)$, or $\left|x_{i}^{\prime}-1-y_{i}\right| \geq t$ in which case $x_{i}^{\prime}=y_{i}-t+1$. Since $y \in V(F)$, there exists $j$ such that $y_{i}+y_{j}=\mathrm{d}\left(B_{i}, B_{j}\right)$. Therefore, since $t \geqslant 2$,

$$
x_{i}^{\prime}+x_{j}^{\prime} \leq\left(y_{i}-t+1\right)+\left(y_{j}+t-1\right)=\mathrm{d}\left(B_{i}, B_{j}\right) .
$$

This concludes the proof.

Note that by Lemma 10 the graph $F$ is connected, since any two vertices are at a finite distance in $F$.

Lemma 11. For all $x \in V(F)$ and $i \in\{1, \ldots, b\}$, it holds that $x_{i}=\mathrm{d}_{F}\left(x, \varphi\left(B_{i}\right)\right)$.

Proof. By Lemma 10, it suffices to prove that $x_{i}=\mathrm{d}^{*}\left(x, \varphi\left(B_{i}\right)\right)$. Since $\mathrm{d}^{*}\left(x, \varphi\left(B_{i}\right)\right)=$ $\max _{j}\left|x_{j}-\mathrm{d}\left(B_{i}, B_{j}\right)\right|$, it follows that $x_{i} \leqslant \mathrm{~d}^{*}\left(x, \varphi\left(B_{i}\right)\right)$. We will now prove that $x_{i} \geqslant$ $\left|x_{j}-\mathrm{d}\left(B_{i}, B_{j}\right)\right|$ for all $j$, which yields the result.

Fix an index $j \neq i$. Since $x_{i}+x_{j} \geq \mathrm{d}\left(B_{i}, B_{j}\right)$, we obtain $x_{i} \geq \mathrm{d}\left(B_{i}, B_{j}\right)-x_{j}$. So it only remains to show that $x_{i} \geqslant x_{j}-\mathrm{d}\left(B_{i}, B_{j}\right)$. Since $x \in V(F)$, there exists $k$ such that $x_{j}+x_{k}=\mathrm{d}\left(B_{j}, B_{k}\right)$. Therefore

$$
\begin{aligned}
x_{j}=\mathrm{d}\left(B_{j}, B_{k}\right)-x_{k} & \leq \mathrm{d}\left(B_{j}, B_{k}\right)-\left(\mathrm{d}\left(B_{i}, B_{k}\right)-x_{i}\right) \\
& \leq x_{i}+\mathrm{d}\left(B_{i}, B_{j}\right) .
\end{aligned}
$$

Thus $x_{i} \geq x_{j}-\mathrm{d}\left(B_{i}, B_{j}\right)$, which concludes the proof.

Lemma 12. The boundary of $F$ is $\left\{\varphi\left(B_{1}\right), \ldots, \varphi\left(B_{b}\right)\right\}$.

Proof. Let $x$ be a boundary vertex of $F$ and $y$ be a witness for $x$. Set $t:=\mathrm{d}_{F}(x, y)$. We first prove that there exists $i \in\{1, \ldots, b\}$ such that $y_{i}=x_{i}+t$. By Lemma 10, there exists $j \in\{1, \ldots, b\}$ such that $t=\left|x_{j}-y_{j}\right|$. If $y_{j}=x_{j}+t$ then we set $i:=j$. If $y_{j}=x_{j}-t$ then since there exists $i$ such that $x_{j}+x_{i}=\mathrm{d}\left(B_{i}, B_{j}\right)$ by (F2), we obtain $x_{i}=\mathrm{d}\left(B_{i}, B_{j}\right)-x_{j}=\mathrm{d}\left(B_{i}, B_{j}\right)-y_{j}-t \leq y_{i}-t$ using (F1). As $y_{i} \leq x_{i}+t$, it follows that $y_{i}=x_{i}+t$. Since $\mathrm{d}_{F}\left(\varphi\left(B_{i}\right), y\right)=y_{i}=x_{i}+t=\mathrm{d}_{F}\left(\varphi\left(B_{i}\right), x\right)+\mathrm{d}_{F}(x, y)$, there is a shortest path between $\varphi\left(B_{i}\right)$ and $y$ that contains $x$. Since $y$ is a witness for $x$, we infer that $x=\varphi\left(B_{i}\right)$. Thus, $\mathscr{B}(F) \subseteq\left\{\varphi\left(B_{1}\right), \ldots, \varphi\left(B_{b}\right)\right\}$.

To finish the proof, we need to show that $\varphi\left(B_{i}\right)$ is a boundary vertex of $F$ for each $i \in\{1,2, \ldots, b\}$. Pick an arbitrary $i$, and let $j$ be such that $B_{j}$ is a witness for $B_{i}$ in $G$ (such a $j$ exists by Lemma 6 ). We show that $\varphi\left(B_{j}\right)$ is also a witness for $\varphi\left(B_{i}\right)$ in $F$. Let $x \in V(F)$ be a neighbour of $\varphi\left(B_{i}\right)$, and suppose that $\mathrm{d}_{F}\left(x, \varphi\left(B_{j}\right)\right)>\mathrm{d}_{F}\left(\varphi\left(B_{i}\right), \varphi\left(B_{j}\right)\right)$. Then there exists a $\varphi\left(B_{j}\right) x$-path of length $\mathrm{d}_{F}\left(x, \varphi\left(B_{j}\right)\right)$ that goes through $\varphi\left(B_{i}\right)$. We already know that $\mathscr{B}(F) \subseteq\left\{\varphi\left(B_{1}\right), \ldots, \varphi\left(B_{b}\right)\right\}$, so that this path must extend to a shortest $\varphi\left(B_{i}\right) \varphi\left(B_{k}\right)$-path for some $k$ by Lemma 6 . Hence, $\mathrm{d}_{G}\left(B_{j}, B_{i}\right)+\mathrm{d}_{G}\left(B_{i}, B_{k}\right)=$ 
$\mathrm{d}_{F}\left(\varphi\left(B_{j}\right), \varphi\left(B_{i}\right)\right)+\mathrm{d}_{F}\left(\varphi\left(B_{i}\right), \varphi\left(B_{k}\right)\right)=\mathrm{d}_{F}\left(\varphi\left(B_{j}\right), \varphi\left(B_{k}\right)\right)=\mathrm{d}_{G}\left(B_{j}, B_{k}\right)$. So in $G$ there must also be a path of length $\mathrm{d}_{G}\left(B_{j}, B_{k}\right)$ going through $B_{i}$. But this implies $B_{i}$ has a neighbour $v$ with $\mathrm{d}_{G}\left(v, B_{j}\right)=\mathrm{d}_{G}\left(B_{i}, B_{j}\right)+1$, which contradicts the assumption that $B_{j}$ is a witness for $B_{i}$ in $G$.

Hence, $\varphi\left(B_{i}\right)$ is a boundary vertex of $F$ for all $i \in\{1,2, \ldots, b\}$. This concludes the proof.

The next theorem summarizes the previous study.

Theorem 13. Let $\mathscr{F}$ be a frame on the points $B_{1}, \ldots, B_{b}$, and suppose that the graph $G$ has frame $\mathscr{F}$. Set $F:=F(\mathscr{F})$. Then the map

$$
\begin{aligned}
\varphi: V(G) & \longrightarrow V(F) \\
v & \longmapsto \varphi(v)=\left(\mathrm{d}_{G}\left(v, B_{1}\right), \ldots, \mathrm{d}_{G}\left(v, B_{b}\right)\right)
\end{aligned}
$$

is an embedding of $G$ into $F$ as an induced subgraph. Moreover, the $\operatorname{set}\left\{\varphi\left(B_{1}\right), \ldots, \varphi\left(B_{b}\right)\right\}$ is precisely the boundary of $F$ and $\mathrm{d}_{G}(u, v)=\mathrm{d}_{F}(\varphi(u), \varphi(v))$ for all vertices $u, v \in V(G)$.

Note that the theorem in particular implies that $F$ has frame $\mathscr{F}$ (if we identify $\varphi\left(B_{i}\right)$ with $\left.B_{i}\right)$.

Proof of Theorem 13. That $\varphi$ is an embedding, and $G$ is an induced subgraph of $F$ follows directly from Lemma 7, Lemma 8 and the definition of $F$. By Lemma 10 and Lemma 7 it follows that $\mathrm{d}_{G}(u, v)=\mathrm{d}^{*}(\varphi(u), \varphi(v))=\mathrm{d}_{F}(\varphi(u), \varphi(v))$. By Lemma 12 the boundary of $F$ is $\left\{\varphi\left(B_{1}\right), \ldots, \varphi\left(B_{b}\right)\right\}$.

\section{The frame of a graph with four boundary vertices and minimum degree at least 2}

In this section we study the graph $F$ corresponding to (the frame of) a connected graph $G$ with four boundary vertices and minimum degree at least 2 (vertices of degree 1 are dealt with later on). In view of Theorem 13, we identify $v \in V(G)$ with $\varphi(v) \in V(F)$ for the ease of exposition.

Let $B_{1}, B_{2}, B_{3}$ and $B_{4}$ be the boundary vertices of $F$ and $G$. By assumption, each of them has degree at least 2 .

Lemma 14. Let $G$ be a graph with minimum degree at least 2 . For every $B \in \mathscr{B}(G)$, there exist two distinct vertices $A_{1}$ and $A_{2}$ in $\mathscr{B}(G) \backslash\{B\}$, such that the shortest $B A_{1}$-path and the shortest $B A_{2}$-path in $G$ are unique, and these two paths only have the vertex $B$ in common.

Proof. We consider two cases.

Case 1: There exists a shortest path between two boundary vertices $A_{1}$ and $A_{2}$ containing $B$. We assert that the path $A_{1} B$ is unique. Assume, to the contrary, that there is another 
shortest path between $A_{1}$ and $B$. Observe that there exist two distinct vertices $x$ and $y$ that are at the same distance from $B$, and at the same distance from $A_{1}$. They also are at the same distance from $A_{2}$. Therefore, the endvertices of an extension of a shortest path between $x$ and $y$ must be distinct from $A_{1}, A_{2}$ and $B$. But these endvertices are boundary vertices, which is a contradiction since there are four boundary vertices in total. Similarly, the path $B A_{2}$ is unique as well.

Note that the $A_{1} B$-path and the $B A_{2}$-path can only have the vertex $B$ in common, since their lengths sum up to the distance between $A_{1}$ and $A_{2}$.

Case 2: The vertex $B$ is not contained in any shortest path between two boundary vertices distinct from $B$. In this case, we assert that the neighbourhood of $B$ induces a clique. Indeed, if $u$ and $v$ are two non-adjacent neighbours of $B$ then extending the shortest path $u B v$ shows that $B$ belongs to a shortest path between two other boundary vertices, a contradiction. Let $u$ and $v$ be two neighbours of $B$. Let $P$ be the extension of the path $u v$ with endvertices $A_{1}$ and $A_{2}$, where $A_{1}$ is closer to $u$.

Let $U:=\left\{s \in N(B): \mathrm{d}\left(A_{1}, s\right)+1=\mathrm{d}\left(A_{1}, B\right)\right\}$. Note that $u \in U$. We assert that $U=\{u\}$. To show this, it suffices to prove that every vertex in $U$ has the same distance to $A_{1}, B$ and $A_{2}$ as well (this is because if $w$ is such that $\mathrm{d}\left(w, A_{1}\right)=\mathrm{d}\left(u, A_{1}\right), \mathrm{d}\left(w, A_{2}\right)=$ $\mathrm{d}\left(u, A_{2}\right)$ and $\mathrm{d}(w, B)=\mathrm{d}(u, B)$ then extending a shortest $u w$-path show that there are at least 5 boundary vertices, a contradiction). Indeed, for $A_{2}$, suppose that $w \in U$. It holds that $\mathrm{d}\left(w, A_{2}\right) \leqslant \mathrm{d}\left(u, A_{2}\right)$, since $N(B)$ induces a clique, and thus $u v \in E$. If the inequality is strict, then there is a path between $A_{1}$ and $A_{2}$ of length $\mathrm{d}\left(A_{1}, w\right)+\mathrm{d}\left(w, A_{2}\right)$, which contradicts that $P$ is a shortest $A_{1} A_{2}$-path.

Now, the shortest path between $u$ and $A_{1}$ is unique, for otherwise there exist two vertices that are at the same distance from $B, A_{1}$ and $A_{2}$, a contradiction. Consequently, the path $B A_{1}$ is unique as well. By symmetry, the path $B A_{2}$ is unique. Again, the paths cannot have any vertex other than $B$ in common.

Lemma 15. Let $G$ be a graph with minimum degree at least 2 and exactly 4 boundary vertices $B_{1}, B_{2}, B_{3}, B_{4}$. Without loss of generality, we can assume that the shortest $B_{1} B_{2^{-}}$, $B_{2} B_{3^{-}}, B_{3} B_{4}$ - and $B_{1} B_{4}$-paths are unique, and that among these paths, those that share an endvertex do not share any other vertex.

Proof. The sought conclusion holds if $\operatorname{diam}(G)=1$, in which case $G$ is $K_{4}$. So we assume throughout the rest of the proof that $\operatorname{diam}(G) \geqslant 2$. Note that if two vertices are at distance $\operatorname{diam}(G)$ of each other, then they are boundary vertices. We split the analysis into two cases.

Suppose first that whenever two vertices are at distance $\operatorname{diam}(G)$ of each other, then there is a unique shortest path between them. Up to relabelling the boundary vertices, we may assume that $\mathrm{d}\left(B_{1}, B_{2}\right)=\operatorname{diam}(G)$. Let $P$ be the unique shortest path between $B_{1}$ and $B_{2}$. Any neighbour of $B_{1}$ that is not on $P$ is at distance at least $\operatorname{diam}(G)$ from $B_{2}$. Thus, any such neighbour is a boundary vertex of $G$ (with witness $B_{2}$ ). Since $B_{1}$ has degree at least 2 , we deduce that there is an edge between $B_{1}$ and, say, $B_{4}$. Note that necessarily $\mathrm{d}\left(B_{2}, B_{4}\right)=\operatorname{diam}(G)$. Similarly, there is an edge between $B_{2}$ and $B_{3}$ (note that $B_{2}$ is not adjacent to $B_{4}$ since $\left.\operatorname{diam}(G) \geqslant 2\right)$ and $\mathrm{d}\left(B_{4}, B_{3}\right)=\operatorname{diam}(G)$. This 
concludes the proof in the case where any two vertices at distance $\operatorname{diam}(G)$ have a unique shortest path between them.

Assume now that there are two vertices of $G$ at distance $\operatorname{diam}(G)$ of each other with at least two distinct shortest paths between them. Up to relabelling the boundary vertices, we may assume that those two vertices are $B_{1}$ and $B_{3}$. By applying Lemma 14 to $B_{1}$ and $B_{3}$, we infer that the shortest paths between $B_{1}, B_{2}$, between $B_{2}, B_{3}$, between $B_{3}, B_{4}$ and between $B_{4}, B_{1}$ are all unique. If there are at least two distinct shortest paths between $B_{2}$ and $B_{4}$, then applying Lemma 14 to each boundary vertex yields the desired conclusion. So we may assume that there is a unique shortest path $P$ between $B_{2}$ and $B_{4}$. Now, the aforementioned unique shortest paths satisfy the statement of the theorem unless they intersect. Assume that the unique shortest paths $P_{12}$ between $B_{1}$ and $B_{2}$, and $P_{23}$ between $B_{2}$ and $B_{3}$ intersect. Since those paths are unique, the neighbour of $B_{2}$ on $P_{12}$ also belongs to $P_{23}$. There is another neighbour $X$ of $B_{2}$, since $G$ has minimum degree at least 2 . Let $x:=\mathrm{d}\left(B_{1}, B_{2}\right), y:=\mathrm{d}\left(B_{3}, B_{2}\right)$ and $z:=\mathrm{d}\left(B_{4}, B_{2}\right)$. By Theorem 13 and the fact that $\operatorname{diam}(G)=\mathrm{d}\left(B_{1}, B_{3}\right) \leq x+y-2$, the position vector of $X$ is $(x, 1, y, z-1)$. Further, we conclude that $\mathrm{d}\left(B_{1}, B_{4}\right)=x+z-1$ and $\mathrm{d}\left(B_{3}, B_{4}\right)=y+z-1$. The path $P$ intersects a shortest path between $B_{1}$ and $B_{3}$ at some vertex $T$. Indeed, since the shortest $B_{1} B_{3^{-}}$ path is not unique, there are two vertices $T$ and $T^{\prime}$ such that $\mathrm{d}\left(T, B_{1}\right)=\mathrm{d}\left(T^{\prime}, B_{1}\right)$ and $\mathrm{d}\left(T, B_{3}\right)=\mathrm{d}\left(T^{\prime}, B_{3}\right)$. Thus, a shortest $T T^{\prime}$-path extends to a shortest path between $B_{2}$ and $B_{4}$, which must be $P$ (since the shortest $B_{2} B_{4}$-path is unique). Let $t:=\mathrm{d}\left(B_{4}, T\right)<z$. Now, $x+z-1=\mathrm{d}\left(B_{1}, B_{4}\right) \leq \mathrm{d}\left(B_{1}, T\right)+t$ and $y+z-1=\mathrm{d}\left(B_{3}, B_{4}\right) \leq \mathrm{d}\left(B_{3}, T\right)+t$. So

$$
x+y-2+2 z \leq \mathrm{d}\left(B_{1}, T\right)+\mathrm{d}\left(B_{3}, T\right)+2 t=\mathrm{d}\left(B_{1}, B_{3}\right)+2 t \leq x+y-2+2 t .
$$

This is a contradiction since $t<z$.

In the remainder of this section we let $\mathrm{P}_{i j}$ be the unique shortest $B_{i} B_{j}$-path. Let us set

$$
\begin{array}{lll}
d_{12}:=\mathrm{d}\left(B_{1}, B_{2}\right), & d_{13}:=\mathrm{d}\left(B_{1}, B_{3}\right), & d_{14}:=\mathrm{d}\left(B_{1}, B_{4}\right), \\
d_{34}:=\mathrm{d}\left(B_{3}, B_{4}\right), & d_{24}:=\mathrm{d}\left(B_{2}, B_{4}\right), & d_{23}:=\mathrm{d}\left(B_{2}, B_{3}\right) .
\end{array}
$$

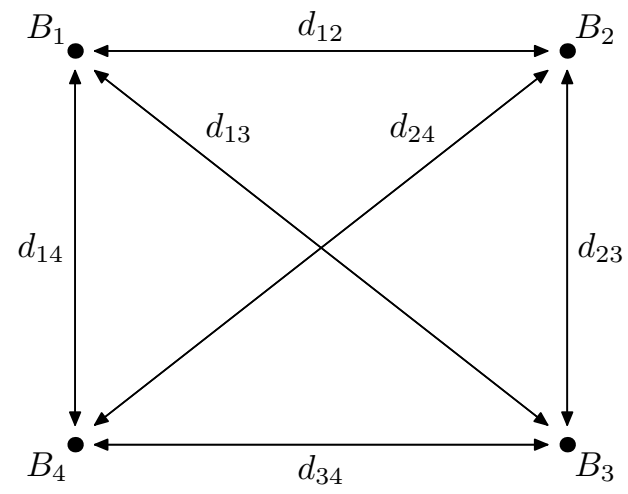

Figure 4: Notations of the distances between the boundary vertices. 
Lemma 16. Without loss of generality, we may assume that one of the following holds:

(i) $d_{12}=d_{34}, d_{14}=d_{23}$, and $d_{13}=d_{24}=d_{12}+d_{14}$;

(ii) $d_{12}=d_{34}, d_{14}=d_{23}$, and $d_{13}=d_{24}=d_{12}+d_{14}-1$;

(iii) $d_{12}=d_{34}, d_{14}=d_{23}, d_{13}=d_{12}+d_{14}$, and $d_{24}=d_{12}+d_{14}-1$;

(iv) $d_{12}=d_{34}, d_{23}=d_{14}+1$, and $d_{13}=d_{24}=d_{12}+d_{14}$.

Proof. Note that the lemma is true if $\operatorname{diam}(G)=1$. So we assume that $\operatorname{diam}(G) \geqslant 2$.

First, we show that $d_{24} \in\left\{d_{12}+d_{14}-1, d_{12}+d_{14}\right\}$. If $B_{1}$ and $B_{2}$ are adjacent, i.e. if $d_{12}=1$, then the triangle inequality ensures that $d_{24} \in\left\{d_{14}-1, d_{14}, d_{14}+1\right\}$. Moreover, $d_{24} \neq d_{14}-1$ for otherwise $P_{14}$ and $P_{12}$ would share the vertex $B_{2}$, thereby contradicting Lemma 15. The situation is analogous if $B_{1}$ and $B_{4}$ are adjacent, i.e. if $d_{14}=1$. So, assume that both $d_{12}$ and $d_{14}$ are greater than 1 . Let $v_{i j}$ be the neighbour of $B_{i}$ on $P_{i j}$. Extending the shortest path between $v_{14}$ and $v_{12}$ (note that they are distinct by the proof of Lemma 14), we obtain a shortest path between $B_{2}$ and $B_{4}$. Indeed, $B_{1}$ cannot be involved since it is adjacent to both $v_{14}$ and $v_{12}$. Further, if $B_{3}$ were involved then for $v=B_{2}$ or $v=B_{4}$ it would follow that the unique shortest $v B_{1}$-path and the unique shortest $v B_{3}$-path share a vertex different from $v$. This would contradict Lemma 15 . This shows that $d_{24} \in\left\{d_{12}+d_{14}, d_{12}+d_{14}-1\right\}$.

Similarly, by considering $d_{34}, d_{23}$, and $v_{34}, v_{32}$, we deduce that $d_{24} \in\left\{d_{34}+d_{23}, d_{34}+\right.$ $\left.d_{23}-1\right\}$. Thus one of the following holds.

(b'.1) $d_{12}+d_{14}=d_{34}+d_{23}$

(b'.2) $d_{12}+d_{14}=d_{34}+d_{23}-1=d_{24} ;$

(b'.3) $d_{12}+d_{14}-1=d_{34}+d_{23}=d_{24}$.

Proceeding analogously with $B_{1}$ and $B_{4}$ yields that $d_{13} \in\left\{d_{34}+d_{14}, d_{34}+d_{14}-1\right\}$ and $d_{13} \in\left\{d_{12}+d_{23}, d_{12}+d_{23}-1\right\}$. Consequently, one of the following holds.

(b.1) $d_{12}+d_{23}=d_{34}+d_{14}$;

(b.2) $d_{12}+d_{23}=d_{34}+d_{14}-1=d_{13}$;

(b.3) $d_{12}+d_{23}-1=d_{34}+d_{14}=d_{13}$.

Both one of (b'.1), (b'.2), (b'.3) and one of (b.1), (b.2), (b.3) hold.

Suppose first that ( $\left.\mathbf{b}^{\prime} . \mathbf{1}\right)$ holds. Then, $d_{12}-d_{34}=d_{23}-d_{14}$. If (b.1) holds then $d_{12}-d_{34}=d_{14}-d_{23}$. So $d_{14}-d_{23}=-\left(d_{14}-d_{23}\right)$, giving that $d_{14}=d_{23}$ and $d_{12}=d_{34}$. Thus, up to swapping $B_{1}$ and $B_{4}$, and swapping $B_{2}$ and $B_{3}$, we are in situation (i), (ii) or (iii). (Note that such a swapping does not spoil Lemma 15.) If (b.2) holds then $d_{12}-d_{34}=d_{14}-d_{23}-1$. So, $2\left(d_{14}-d_{23}\right)=1$, i.e. $d_{14}=d_{23}+1 / 2$. But this cannot be since both $d_{14}$ and $d_{23}$ are integers. The situation when (b.3) holds is similar. 
Assume now that (b'.2) holds. Thus, $d_{12}-d_{34}=d_{23}-d_{14}-1$. If (b.1) holds then $d_{12}-d_{34}=d_{14}-d_{23}$. So $2\left(d_{14}-d_{23}\right)=1$, a contradiction. If (b.2) holds then $d_{12}-d_{34}=d_{14}-d_{23}-1$. So $d_{23}=d_{14}$ and $d_{34}=d_{12}+1$, and hence up to relabelling we are in situation (iv). Last, if (b.3) holds then $d_{12}-d_{34}=d_{14}-d_{23}+1$. So $d_{23}=d_{14}+1$ and $d_{12}=d_{34}$, and hence we are in situation (iv).

Finally, the situation when (b'.3) holds is completely analogous to the situation when (b'.2) holds.

Theorem 17. Let $G$ be a graph with minimum degree at least 2 and exactly four boundary vertices. Let $F$ be the graph corresponding to its frame as constructed in Definition 9. Then $F$ is isomorphic to either $N_{d_{12} \times d_{14}}, X_{d_{12} \times d_{14}}, T_{d_{12} \times d_{14}}$ or $D_{d_{12} \times d_{14}}$.

For convenience we split the theorem into several lemmas.

\section{Lemma 18.}

(i) If $d_{12}=d_{34}, d_{14}=d_{23}$, and $d_{13}=d_{24}=d_{12}+d_{14}$ then $F$ is isomorphic to $N_{d_{12} \times d_{14}}$.

(ii) If $d_{12}=d_{34}, d_{23}=d_{14}+1$, and $d_{13}=d_{24}=d_{12}+d_{14}$ then $F$ is isomorphic to $T_{d_{12} \times d_{14}}$.

Proof. For non-negative integers $x$ and $y$ let us set

$$
\begin{aligned}
v_{x, y} & :=\left(x+y, d_{12}-x+y, d_{12}+d_{14}-x-y, d_{14}+x-y\right) \quad \text { and } \\
w_{x, y} & :=\left(x+y+1, d_{12}-x+y, d_{12}+d_{14}-1-x-y, d_{14}+x-y\right) .
\end{aligned}
$$

(i). Observe that $v_{x, y}$ is the position vector for the vertex $(x, y)$ in $N_{d_{12} \times d_{14}}$ for every $x \in\left\{0,1, \ldots, d_{12}\right\}$ and every $y \in\left\{0,1, \ldots, d_{14}\right\}$. Further, $w_{x, y}$ is the position vector of $\left(x+\frac{1}{2}, y+\frac{1}{2}\right)$ in $N_{d_{12} \times d_{14}}$ if $x \in\left\{0,1, \ldots, d_{12}-1\right\}$ and $y \in\left\{0,1, \ldots, d_{14}-1\right\}$. Therefore these vectors satisfy the requirements $(\mathbf{F} 1)$ and $($ F2), so that they form the vertices of an induced copy of $N_{d_{12} \times d_{14}}$ inside $F$.

Now pick $u=\left(u_{1}, u_{2}, u_{3}, u_{4}\right) \in V(F)$. We wish to show that $u \in V\left(N_{d_{12} \times d_{14}}\right)$, which will give that $F$ indeed coincides with $N_{d_{12} \times d_{14}}$. We know that $u_{1}+u_{2} \geq d_{12}$. First suppose that $u_{1}+u_{2}=d_{12}$. Then $u$ lies on a $B_{1} B_{2}$-path of length $d_{12}$. Since there is only one such path (by Lemma 15), namely $\mathrm{P}_{12}=v_{0,0} v_{1,0} \ldots v_{d_{12}, 0}$, we deduce that $u \in V\left(N_{d_{12} \times d_{14}}\right)$. Let us thus assume that $u_{1}+u_{2}>d_{12}$. Similarly, we can suppose that $u_{2}+u_{3}>d_{14}, u_{3}+u_{4}>d_{12}$, and $u_{1}+u_{4}>d_{14}$. Observe that, by (F2),

$$
u_{1}+u_{3}=u_{2}+u_{4}=d_{12}+d_{14}
$$

Now assume that $u_{1}+u_{2}-d_{12}$ is even, and set $y:=\left(u_{1}+u_{2}-d_{12}\right) / 2$ and $x:=u_{1}-y$. Hence, $u_{1}=x+y$ and $u_{2}=d_{12}-x+y$, so that by (1) we deduce that $u_{3}=d_{12}+d_{14}-x-y$ and $u_{4}=d_{14}+x-y$. Because $d_{14}+2 x=u_{1}+u_{4}>d_{14}$ and $2 d_{12}+d_{14}-2 x=u_{2}+u_{3}>d_{14}$, it follows that $0<x<d_{12}$. Similarly, we obtain $0<y<d_{14}$ since $d_{12}+2 y=u_{1}+u_{2}>d_{12}$ and $d_{12}+2 d_{14}-2 y=u_{3}+u_{4}>d_{12}$. Consequently, $u=v_{x, y} \in V\left(N_{d_{12} \times d_{14}}\right)$ if $u_{1}+u_{2}-d_{12}$ is even. 
Let us assume now that $u_{1}+u_{2}-d_{12}$ is odd. We set $y:=\left(u_{1}+u_{2}-d_{12}-1\right) / 2$ and $x:=u_{1}-y-1$. Thus, $u_{1}=x+y+1$ and $u_{2}=d_{12}-x+y$. In addition, (1) implies that $u_{3}=d_{12}+d_{14}-1-x-y$ and $u_{4}=d_{14}+x-y$. Because $d_{14}+2 x+1=u_{1}+u_{4}>d_{14}$ and $2 d_{12}+d_{14}-1-2 x=u_{2}+u_{3}>d_{14}$, we obtain $0 \leq x<d_{12}$. And because $d_{12}+2 y+1=$ $u_{1}+u_{2}>d_{12}$ and $d_{12}+2 d_{14}-1-2 y=u_{3}+u_{4}>d_{12}$, we obtain $0 \leq y<d_{14}$. Hence, $u=w_{x, y} \in V\left(N_{d_{12} \times d_{14}}\right)$ when $u_{1}+u_{2}-d_{12}$ is odd.

So $F$ and $N_{d_{12} \times d_{14}}$ indeed coincide.

(ii). The points in

$$
\begin{aligned}
W:= & \left\{v_{x, y}: 0 \leq x<d_{12} \text { and } 0 \leq y \leq d_{14}\right\} \\
& \cup\left\{w_{x, y}: 0 \leq x<d_{12} \text { and } 0 \leq y<d_{14}\right\} \\
& \cup\left\{\left(d_{12}-1+i, i, d_{14}+1-i, d_{12}+d_{14}-i\right): 0<i \leqslant d_{14}\right\} \\
& \cup\left\{\left(d_{12}, 0, d_{14}+1, d_{12}+d_{14}\right),\left(d_{12}+d_{14}, d_{14}+1,0, d_{12}\right)\right\}
\end{aligned}
$$

satisfy the requirements $(\mathbf{F} 1)$ and $(\mathbf{F} 2)$. So $W \subseteq V(F)$. Also note that $W$ is precisely the set of position vectors of $T_{d_{12} \times d_{14}}$, so that $T_{d_{12} \times d_{14}}$ is an induced subgraph of $F$.

Now pick $u \in V(F)$. Analogously as before, we may assume that $u_{1}+u_{2}>d_{12}$, $u_{2}+u_{3}>d_{14}+1, u_{3}+u_{4}>d_{12}$, and $u_{1}+u_{4}>d_{14}$. Moreover, $u_{1}+u_{3}=u_{2}+u_{4}=d_{12}+d_{14}$. Suppose that $u_{1}+u_{2}-d_{12}$ is even. Set $y:=\left(u_{1}+u_{2}-d_{12}\right) / 2$ and $x:=u_{1}-y$. Then $u_{1}=x+y$ and $u_{2}=d_{12}-x+y$. It thus follows that $u=v_{x, y}$. In addition, since $u_{1}+u_{2}>d_{12}, u_{2}+u_{3}>d_{14}+1, u_{3}+u_{4}>d_{12}$, and $u_{1}+u_{4}>d_{14}$, it follows that $0<x<d_{12}$ and $0<y<d_{14}$. Hence, $u \in V\left(T_{d_{12} \times d_{14}}\right)$ if $u_{1}+u_{2}-d_{12}$ is even.

Now suppose that $u_{1}+u_{2}-d_{12}$ is odd. Set $y:=\left(u_{1}+u_{2}-d_{12}-1\right) / 2$ and $x:=u_{1}-y$. Analogously to before, we infer that $u=w_{x, y}$ with $0 \leq x<d_{12}$ and $0 \leq y<d_{14}$ if $u_{1}+u_{2}-d_{12}$ is odd.

Thus $F$ coincides with $T_{d_{12} \times d_{14}}$ as required.

Lemma 19. If $d_{12}=d_{34}, d_{14}=d_{23}, d_{13}=d_{12}+d_{14}$, and $d_{24}=d_{12}+d_{14}-1$ then $F$ is isomorphic to $D_{d_{12} \times d_{14}}$.

Proof. Recall that $B_{1}=\left(0, d_{12}, d_{12}+d_{14}, d_{14}\right), B_{2}=\left(d_{12}, 0, d_{14}, d_{12}+d_{14}-1\right), B_{3}=$ $\left(d_{12}+d_{14}, d_{14}, 0, d_{12}\right)$, and $B_{4}=\left(d_{14}, d_{12}+d_{14}-1, d_{12}, 0\right)$. For $x \in\left\{1,2, \ldots, d_{12}\right\}$ and $y \in\left\{0,1, \ldots, d_{14}-1\right\}$ let us set

$$
v_{x, y}:=\left(x+y, d_{12}-x+y, d_{12}+d_{14}-(x+y), d_{14}-1+x-y\right) .
$$

For $x \in\left\{0,1, \ldots, d_{12}-1\right\}$ and $y \in\left\{1,2, \ldots, d_{14}\right\}$ let us set

$$
w_{x, y}:=\left(x+y, d_{12}-1-x+y, d_{12}+d_{14}-(x+y), d_{14}+x-y\right) .
$$

First, note that $B_{2}=v_{d_{12}, 0}$ and $B_{4}=w_{0, d_{14}}$ but neither $B_{1}$ nor $B_{3}$ corresponds to any of the points $v_{x, y}$ or $w_{x, y}$. Observe also that the points $v_{x, y}$ and $w_{x, y}$ satisfy the criteria (F1) and (F2), and that they are the position vectors of the vertices of $D_{d_{12} \times d_{14}}$ other than $B_{1}$ 
and $B_{3}$. Hence, together with $B_{1}$ and $B_{3}$, they form an induced copy of $D_{d_{12} \times d_{14}}$ inside $F$.

Now let $u=\left(u_{1}, u_{2}, u_{3}, u_{4}\right) \in V(F)$ be arbitrary. First, suppose that $u_{1}+u_{2}=$ $d_{12}$. Then, $u$ is on the unique shortest path $\mathrm{P}_{12}$ between $B_{1}$ and $B_{2}$. Notice that $B_{1} v_{1,0} \ldots v_{d_{12}-1,0} B_{2}$ is a $B_{1} B_{2}$-path of length $d_{12}$. Hence it equals $\mathrm{P}_{12}$. It follows that $u \in D_{d_{12} \times d_{14}}$. Thus, we can suppose that $u_{1}+u_{2}>d_{12}$. Similarly, we may assume $u_{1}+u_{4}>d_{14}, u_{2}+u_{3}>d_{14}$, and $u_{3}+u_{4}>d_{12}$. Notice that

$$
u_{1}+u_{3}=d_{12}+d_{14}, \quad u_{2}+u_{4}=d_{12}+d_{14}-1
$$

by (F2).

Suppose that $u_{1}+u_{2}-d_{12}$ is even. Let us write $y=\left(u_{1}+u_{2}-d_{12}\right) / 2$ and $x=u_{1}-y$. Then, $u_{1}=x+y$ and $u_{2}=d_{12}-x+y$. Further, using (2), we infer that $u$ is in fact of the same form as $v_{x, y}$ for some $x$ and $y$. It remains to be checked that $0<x<d_{12}$ and $0 \leq y<d_{14}$. Since $u_{2}+u_{3}=2 d_{12}+d_{14}-2 x>d_{14}$ and $u_{1}+u_{4}=d_{14}-1+2 x>d_{14}$, it follows that $0<x<d_{12}$. Since $u_{1}+u_{2}=d_{12}+2 y>d_{12}$ and $u_{3}+u_{4}=d_{12}+2 d_{14}-1+2 y>d_{12}$, it follows that $0<y<d_{14}$. Thus, $u \in V\left(D_{d_{12} \times d_{14}}\right)$ if $u_{1}+u_{2}-d_{12}$ is even.

Now suppose that $u_{1}+u_{2}-d_{12}$ is odd. Set $y:=\left(u_{1}+u_{2}-d_{12}+1\right) / 2$ and $x:=u_{1}-y$. Note that $u_{1}=x+y, u_{2}=d_{12}-1-x+y$, so that by (2) the vertex $u$ is of the same form as $w_{x, y}$ for this choice of $x$ and $y$. Again it remains to be seen that $0<x<d_{12}$ and $0<y<d_{14}$. Since $u_{2}+u_{3}=2 d_{12}+d_{14}-1-2 x>d_{14}$ and $u_{1}+u_{4}=d_{14}+2 x>d_{14}$, it follows that indeed $0<x<d_{12}$. Further, $0<y<d_{14}$ because $u_{1}+u_{2}=d_{12}-1+2 y>d_{12}$ and $u_{3}+u_{4}=d_{12}+2 d_{14}+2 y>d_{12}$. Thus, we conclude that $u \in V\left(D_{d_{12} \times d_{14}}\right)$ if $u_{1}+u_{2}-d_{12}$ is odd.

Therefore, $F$ coincides with $D_{d_{12} \times d_{14}}$.

Lemma 20. If $d_{12}=d_{34}, d_{14}=d_{23}$, and $d_{13}=d_{24}=d_{12}+d_{14}-1$ then $F$ is isomorphic to $X_{d_{12} \times d_{14}}$.

Proof. By the symmetry of the roles played by $d_{12}$ and $d_{14}$, we may assume that $d_{12} \geqslant d_{14}$ (recall that $X_{d_{12} \times d_{14}}=X_{d_{14} \times d_{12}}$ ). First, note that if $d_{12}=d_{14}=1$ then $F$ and $G$ are necessarily isomorphic to $K_{4}=X_{1 \times 1}$. Thus, suppose that $d_{12} \geqslant 2$. This time set

$$
v_{x, y}:=\left(x+y, d_{12}-1-x+y, d_{12}+d_{14}-1-x-y, d_{14}+x-y\right)
$$

for $0 \leqslant x \leqslant d_{12}-1$ and $1 \leqslant y \leqslant d_{14}-1$, and

$$
w_{x, y}:=\left(x+y+1, d_{12}-1-x+y, d_{12}+d_{14}-2-x-y, d_{14}+x-y\right)
$$

for $0 \leqslant x \leqslant d_{12}-2$ and $0 \leqslant y \leqslant d_{14}-1$. Then $v_{x, y}$ satisfies (F1) and (F2) if $0 \leq x \leq d_{12}-1$ and $1 \leq y \leq d_{14}-1$. Furthermore, $w_{x, y}$ satisfies (F1) and (F2) if $0 \leq x \leq d_{12}-2$ and $0 \leq y \leq d_{14}-1$. In addition, together with $B_{1}, B_{2}, B_{3}$, and $B_{4}$, these vertices induce a copy of $X_{d_{12} \times d_{14}}$ in $F$.

Now pick $u \in V(F)$. We can again assume that $u_{1}+u_{2}>d_{12}, u_{1}+u_{4}>d_{14}$, $u_{2}+u_{3}>d_{14}$, and $u_{3}+u_{4}>d_{12}$. Consequently, $u_{1}+u_{3}=u_{2}+u_{4}=d_{12}+d_{14}-1$. 
If $u_{1}+u_{2}-d_{12}+1$ is even, then set $y:=\left(u_{1}+u_{2}-d_{12}+1\right) / 2$ and $x:=u_{1}-y$. So $u=v_{x, y}$ for some $x \in\left\{1,2, \ldots, d_{12}-2\right\}$ and $y \in\left\{1,2, \ldots, d_{14}-1\right\}$. If $u_{1}+u_{2}-d_{12}+1$ is odd, then we set $y:=\left(u_{1}+u_{2}-d_{12}\right) / 2$ and $x:=u_{1}-y$. So $u=w_{x, y}$ for some $x \in\left\{0,1, \ldots, d_{12}-2\right\}$ and $y \in\left\{1,2, \ldots, d_{14}-2\right\}$.

Thus $F=X_{d_{12} \times d_{14}}$ as required.

\section{The proof of Theorem 4}

Notice that if $G=(V, E)$ is a graph, $v \in V$ and $N(v)=\{u\}$, then $v \in \mathscr{B}(G)$ and

$$
\mathscr{B}(G) \backslash\{v\} \subseteq \mathscr{B}(G \backslash\{v\}) \subseteq(\mathscr{B}(G) \cup\{u\}) \backslash\{v\} .
$$

Thus, if we remove a vertex of degree 1 from a graph with four boundary vertices then we either end up with a graph with exactly three boundary vertices, or with another graph with four boundary vertices. Let $G$ be an arbitrary graph with four boundary vertices. By repeatedly removing vertices of degree 1 , we infer that $G$ is either obtained from a graph with three boundary vertices by attaching a path to a non-boundary vertex, or from a graph with four boundary vertices and minimum degree at least 2 by attaching paths to the boundary vertices. By Theorem 1, the first case corresponds precisely to parts (i), (ii) and (iii) of Theorem 4.

In the rest of the proof we therefore assume that $G$ has four boundary vertices and minimum degree at least 2. Let $F$ be the frame-graph of $G$. By Theorem 17, we know that $F$ is isomorphic to either $N_{a \times c}, X_{a \times c}, T_{a \times c}$ or $D_{a \times c}$ for some integers $a$ and $c$.

The following observation follows immediately from Theorem 13, which implies that $\mathrm{d}_{F}(u, v)=\mathrm{d}_{G}(u, v)$ for all $u, v \in V(G)$.

Corollary 21. Let $u, v \in V(G)$ and let $F$ be the frame-graph of $G$. If there is a unique shortest path $P$ in $F$ between $u$ and $v$, then $P \subseteq G$.

Repeated applications of Corollary 21 yield the following.

- If $F=X_{a \times c}$ then $G=F$;

- if $F=D_{a \times c}$ then either $G=D_{a \times c}$ or $G=L_{a \times c}$;

- if $F=N_{a \times c}$ then $V_{a \times c}^{0} \subseteq V(G)$ and $V_{a \times c}^{1} \cap V(G)$ is axis slice convex; and

- if $F=T_{a \times c}$ then $\mathrm{P}_{23}, V_{a \times(c+1)}^{1} \subseteq V(G)$ and $V(G) \cap V_{a \times(c+1)}^{0}$ is axis slice convex.

Suppose that $F=N_{a \times c}$ and $V(G)=V_{a \times c}^{0} \cup\left(W \cap V_{a \times c}^{1}\right)$ for some axis slice convex set $W$. Then a vertex $v \in V(G) \backslash\left\{B_{1}, B_{2}, B_{3}, B_{4}\right\}$ is not a boundary vertex of $G$, since there is a shortest path to any vertex $u \in V(G)$ (that is also a shortest path in $F$ ), and the path can be extended to a path between $B_{i}$ and $B_{j}$ for some $i$ and $j$.

The case where $F=T_{a \times c}$ can be dealt with similarly. This concludes the proof of Theorem 4. 


\section{References}

[1] J. Cáceres, C. Hernando, M. Mora, I. M. Pelayo, M. L. Puertas, and C. Seara. On geodetic sets formed by boundary vertices. Discrete Math., 306(2):188-198, 2006.

[2] G. Chartrand, D. Erwin, G. L. Johns, and P. Zhang. Boundary vertices in graphs. Discrete Math., 263(1-3):25-34, 2003.

[3] G. Chartrand, D. Erwin, G. L. Johns, and P. Zhang. On boundary vertices in graphs. J. Combin. Math. Combin. Comput., 48:39-53, 2004.

[4] Y. Hasegawa and A. Saito. Graphs with small boundary. Discrete Math., 307:18011807, 2007.

[5] C. Hernando, M. Mora, I. M. Pelayo, and C. Seara. Some structural, metric and convex properties on the boundary of a graph. In Proceedings of the Fifth Cracow Conference on Graph Theory (USTRON '06), volume 24, pages 203-209, 2006.

[6] T. Müller, A. Pór, and J.-S. Sereni. Lower bounding the boundary of a graph in terms of its maximum or minimum degree. Discrete Math., 308(24):6581-6583, 2008. 